\title{
The pharmacokinetics and pharmacodynamics of
}

\section{warfarin in combination with}

\section{ambrisentan in healthy}

\section{volunteers}

\author{
Gennyne Walker, Arun Mandagere, Christopher Dufton \& \\ Jürgen Venitz ${ }^{1}$
}

Gilead Sciences Inc., Westminster, CO and 'Virginia Commonwealth University, Richmond, VA, USA

\author{
Correspondence \\ Gennyne Walker, PhD, Gilead Sciences \\ Inc., 3333 Walnut Street, Boulder, CO \\ 80301, USA. \\ Tel: +17208878650 \\ Fax: +17208878680 \\ E-mail: gennyne.walker@gilead.com
}

\section{Keywords}

ambrisentan, endothelin receptor antagonist, International Normalized Ratio, pharmacodynamics, pharmacokinetics, pulmonary arterial hypertension, R-warfarin, S-warfarin

\section{Received}

17 May 2008

Accepted

1 February 2009

\section{WHAT IS ALRADY KNOWN ABOUT \\ THIS SUBJECT}

-Warfarin anticoagulation therapy is commonly administered to patients with pulmonary arterial hypertension (PAH) to reduce the risk of thrombosis and embolism. - Ambrisentan is an orally active, propanoic acid-based endothelin receptor antagonist that is likely to be co-administered with warfarin to $\mathrm{PAH}$ patients.

- Concomitant therapies that induce or inhibit warfarin metabolism may alter the systemic exposure of warfarin and significantly alter clotting time, as measured by prothrombin time and International Normalized Ratio, thereby requiring substantial adjustments to warfarin dose.

\section{WHAT THIS STUDY ADDS}

- This study was undertaken in healthy subjects to identify any potential drug interactions between ambrisentan and warfarin.

- Multiple doses of ambrisentan had no clinically relevant effects on the pharmacokinetics or pharmacodynamics of warfarin; conversely, a single dose of warfarin had no clinically relevant effects on the steady-state pharmacokinetics of ambrisentan.

- Therefore, co-administration of ambrisentan with warfarin should not require adjustment of the therapeutically effective dosing regimen for either drug.

\section{AIMS}

Ambrisentan is an oral, propanoic acid-based endothelin receptor antagonist often co-administered with warfarin to patients with pulmonary arterial hypertension. The aim of this study was to evaluate the potential for ambrisentan to affect warfarin pharmacokinetics and pharmacodynamics.

\section{METHODS}

In this open-label cross-over study, 22 healthy subjects received a single dose of racemic warfarin $25 \mathrm{mg}$ alone and after 8 days of ambrisentan $10 \mathrm{mg}$ once daily. Assessments included exposure $\left(A \cup C_{0-\text { last }}\right)$ and maximum plasma concentration $\left(C_{\max }\right)$ for $\mathrm{R}$ - and S-warfarin, and International Normalized Ratio maximum observed value $\left(I_{N R_{\text {max }}}\right)$ and area under the curve $\left(I N R R_{A \cup C(0-\text { last })}\right)$. The effects of warfarin on ambrisentan steady-state pharmacokinetics and the safety of ambrisentan/warfarin co-administration were assessed. Data are presented as geometric mean ratios.

\section{RESULTS}

Ambrisentan had no significant effects on the $\mathrm{AUC}_{0 \text {-last }}$ of R-warfarin [104.7; 90\% confidence interval (CI) 101.7, 107.7) or S-warfarin (101.6; $90 \% \mathrm{Cl} 98.4,105.0)$. Similarly, ambrisentan had no significant effects on the $C_{\max }$ of R-warfarin $(91.6 ; 90 \% \mathrm{Cl} 86.2,97.4)$ or S-warfarin (89.9; $90 \% \mathrm{Cl} 84.8,95.3)$. Consistent with these observations, little pharmacodynamic change was observed for INR $\mathrm{Imax}_{\max }(85.3 ; 90 \% \mathrm{Cl} 82.4$, 88.2) or INR $\mathrm{AUC}_{\text {(0-last) }}(93.0 ; 90 \% \mathrm{Cl} 90.8,95.3)$. In addition, Coadministration of warfarin did not alter ambrisentan steady-state pharmacokinetics. Adverse events were infrequent, and there were no bleeding adverse events.

\section{CONCLUSIONS}

Multiple doses of ambrisentan had no clinically relevant effects on the pharmacokinetics and pharmacodynamics of a single dose of warfarin. Therefore, significant dose adjustments of either drug are unlikely to be required with co-administration. 


\section{Introduction}

Pulmonary arterial hypertension (PAH) is a serious and life-threatening disease characterized by progressive vasoconstriction, vascular remodelling and in situ thrombosis $[1,2]$. Disease progression is associated with significant increases in pulmonary vascular resistance and sustained elevations in pulmonary artery pressure, which ultimately lead to right ventricular failure and death $[1,3]$.

Prothrombotic changes, including increased platelet activity, are characteristic of PAH [2]; therefore, anticoagulants such as warfarin are commonly administered to prevent thrombus formation in this patient population [4]. The pharmacodynamic response to warfarin includes increased prothrombin time (PT), which is often expressed as an International Normalized Ratio (INR) [5]. Warfarin is administered as a racemic mixture of $\mathrm{R}(+)$ and $\mathrm{S}(-)$ enantiomers, and the S-enantiomer is approximately two- to fivefold more potent than the R-enantiomer with respect to coagulation activity [6]. Both warfarin enantiomers are metabolized in the liver by cytochrome P450 (CYP) isoenzymes: R-warfarin is primarily metabolized by CYP1A2 and CYP3A4, whereas S-warfarin is primarily metabolized by CYP2C9 [7]. Concomitant therapies that alter CYP enzyme activities may alter the pharmacokinetic exposure of warfarin and significantly affect clotting time as measured by INR. Decreased INR could potentially lead to a higher risk of thromboembolism, while increased INR could result in significant bleeding episodes.

Endothelin receptor antagonists (ERAs) have emerged as a promising class of therapeutic agents for PAH [4]. Bosentan and sitaxsentan are sulphonamide-based ERAs that have proven effective in the treatment of $\mathrm{PAH}[8,9]$; however, both drugs have been shown to alter warfarin metabolism by affecting the activity of CYP3A4 and CYP2C9. Ambrisentan is a once-daily, orally active, propanoic acid-based, receptor subtype $A\left(E T_{A}\right)$ selective ERA that is approved for the treatment of PAH. Ambrisentan has been shown to improve exercise capacity, delay time to clinical worsening, reduce disease symptoms, and improve cardiopulmonary haemodynamics in patients with PAH [10-12]. Unlike bosentan and sitaxsentan, ambrisentan does not induce or inhibit CYP2C9 or CYP3A4 and is therefore unlikely to have drug-drug interactions with warfarin and other CYP-metabolized drugs [11]. This study was undertaken to evaluate the effects of multiple doses of ambrisentan on the pharmacokinetics and pharmacodynamics of a single racemic dose of warfarin, as well as to assess the effects of warfarin on the steady-state pharmacokinetics of ambrisentan. In addition, the safety and tolerability of ambrisentan and warfarin co-administration were evaluated.

\section{Methods}

\section{Subjects}

Men and women aged $18-55$ years with a body mass index of 18.5-29.9, weight $\geq 50 \mathrm{~kg}$, and in good health were eligible for this study. Determination of health was based on a comprehensive pre-study examination that included medical history, physical examination, electrocardiogram (ECG) and clinical laboratory tests. Subjects were required to be nontobacco users, and agreed to discontinue the intake of caffeine, alcohol, grapefruit products, liver, and leafy green vegetables. At screening, subjects were also required to have PT, INR and partial thromboplastin time values within normal limits, serum aspartate aminotransferase and alanine aminotransferase concentrations $<1 \mathrm{x}$ the upper limit of normal, and negative laboratory results for human immunodeficiency virus, hepatitis B and hepatitis C. Female subjects were required to be surgically sterile by hysterectomy and/or bilateral oophorectomy.

Subjects were excluded if they had current evidence or history of alcohol dependence, bleeding disorders, sensitivity to warfarin or ambrisentan, or significant allergies. Subjects were also excluded if they had donated or received blood products within 30 days prior to the first dose of study drug, or had received any medication, herbal remedies, food or alcohol that were known to interfere with CYP enzyme metabolism within 2 weeks prior to the first dose of study drug. All participants provided written informed consent. The study was reviewed and approved by Aspire Independent Review Board (San Diego, CA, USA).

\section{Study design/treatments}

This was a Phase 1, open-label, fixed-sequence, two-way, crossover, single-centre study to evaluate the potential for a pharmacokinetic and/or pharmacodynamic interaction of multiple doses of ambrisentan with a single dose of warfarin in healthy subjects (Figure 1). Following a screening period of 21 days, subjects were administered a single dose of racemic warfarin 25 mg (Bristol-Myers Squibb, Princeton, NJ, USA) on day 1 . On days 5 to 16 , subjects received a single daily dose of ambrisentan $10 \mathrm{mg}$ (Gilead Sciences, Inc., formerly Myogen, Inc., Westminster, CO, USA). On day 13 , subjects were administered a single 10-mg dose of ambrisentan together with a single 25-mg dose of warfarin. All study medications were administered under fasted conditions and under the supervision of clinical study site personnel. This study was performed in compliance with the principles of Good Clinical Practice and the Declaration of Helsinki.

\section{Pharmacokinetics and pharmacodynamics}

Blood samples for the pharmacokinetic assessments of $\mathrm{R}$ - and S-warfarin were collected at the following times relative to the day 1 and day 13 warfarin doses: predose ( $\leq 30$ min prior), and $0.5,1,2,3,4,6,8,10,12,18,24,36,48,72$ 


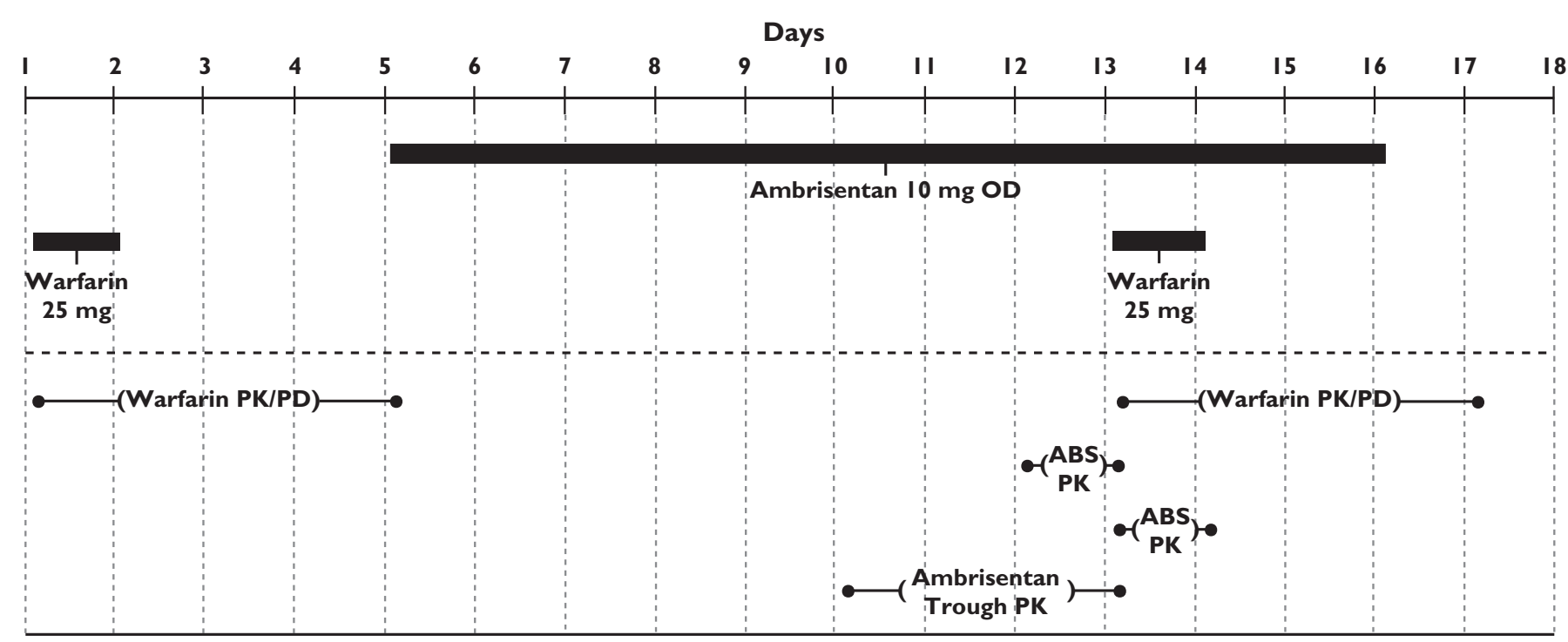

\section{Figure 1}

Study dosing regimen and blood sampling schedule. Single doses of racemic warfarin $25 \mathrm{mg}$ were administered on days 1 and 13 , and ambrisentan $10 \mathrm{mg}$ was administered once daily from day 5 to day 16. OD, once daily; PK, pharmacokinetics; PD, pharmacodynamics; ABS, ambrisentan

and $96 \mathrm{~h}$ postdose. Blood samples for ambrisentan pharmacokinetic assessments were collected relative to the day 12 and day 13 ambrisentan doses: predose $(\leq 30 \mathrm{~min}$ prior), and $0.5,1,2,3,4,6,8,10,12,18$ and $24 \mathrm{~h}$ postdose. Steady-state ambrisentan concentrations were determined from samples collected at trough (predose) on days 10 to 13 . Blood samples were immediately mixed by gentle inversion of the tube eight to 10 times and placed in an ice bath. Within $30 \mathrm{~min}$ of collection, samples were centrifuged at $4^{\circ} \mathrm{C}$ at a minimum of $1500 \mathrm{~g}$ for $15 \mathrm{~min}$ until cells and plasma were well separated. Samples were divided into two aliquots and frozen at $-20^{\circ} \mathrm{C}$ until analysis. Plasma concentrations of R- and S-warfarin were determined by a validated liquid chromatography tandem mass spectrometry (LC/MS/MS) method, with a lower limit of quantification of $1.0 \mathrm{ng} \mathrm{ml}^{-1}$ [13]. Plasma concentrations of ambrisentan were determined by a validated LC/MS/MS method with a lower limit of quantification of $5.0 \mathrm{ng} \mathrm{ml}^{-1}$. The precision of the ambrisentan assay was $<15 \%$ as indicated by the percent coefficient of variation and the accuracy of the assay was within $15 \%$ of the actual value for ambrisentan. Pharmacokinetic analyses were performed using WinNonlin ${ }^{\mathrm{TM}}$ Professional Network Edition, Version 4.0.1 (Pharsight Corp, Palo Alto, CA, USA). Estimated plasma pharmacokinetic parameters for ambrisentan and warfarin enantiomers included area under the concentration-time curve from time 0 to last measurement $\left(A \cup C_{0 \text {-last }}\right.$ ), maximum plasma concentration $\left(C_{\max }\right)$ and time to $C_{\max }$ $\left(t_{\max }\right)$. Minimum plasma concentrations $\left(C_{\min }\right)$ were also determined for the steady-state pharmacokinetic analysis of ambrisentan. Prothrombin times, presented as INR, were characterized using area under the effect-time curve from time 0 to the last measurable concentration (INR $\left.\operatorname{Auc}_{\text {A }(0 \text { last })}\right)$, maximum observed value $\left(I N R_{\max }\right)$ and time to reach maximum observed value $\left(I N R_{\text {tmax }}\right)$.

\section{Safety}

The safety and tolerability of ambrisentan and warfarin co-administration was assessed through adverse events (AEs), vital signs, clinical laboratory evaluations and 12-lead ECG. AEs were monitored continuously during the study. Clinical laboratory parameters (including blood chemistry, haematology and urinalysis) and 12-lead ECG were assessed during the prestudy and poststudy examinations.

\section{Statistical methods}

Participants who received at least one dose of study drug were included in the safety assessment (intent-to-treat population). All subjects with plasma concentration data were included in the pharmacokinetic full analysis population. All subjects who received all doses of study drug and had sufficient blood samples and assessments to determine INR $R_{A u c(0-\text { last), }} I N R_{\max }$ and $I N R_{t_{\max }}$ were included in the pharmacodynamic evaluable population.

Pharmacokinetic and pharmacodynamic parameters are presented as arithmetic means and standard deviations, except for $t_{\max }$ values, which are presented as medians with ranges. Values for INR AUC(0-last) $_{\text {w }}$ were calculated using the trapezoidal rule.

To assess the effect of concomitant ambrisentan dosing on warfarin pharmacokinetics and pharmacodynamics, individual within-subject pair-wise differences of day 13 (warfarin + ambrisentan) vs. day 1 (warfarin alone) were summarized for the natural logarithms of $C_{\max }$ and $A \cup C_{0 \text {-last }}$ for R- and S-warfarin, and the natural logarithms of $I N R_{\max }$ and INR $\mathrm{IUCl}_{\text {A-last). }}$ The comparisons of day $13 /$ day 1 
$I N R_{\max }$ and $I N R_{\text {Auc(0-last) }}$ estimates were prospectively defined as the primary assessments of an interaction. All pharmacokinetic and pharmacodynamic comparisons were reported as geometric mean ratios (GMRs) with twosided confidence intervals $(\mathrm{Cl} ; 90 \% \mathrm{Cl}$ for pharmacokinetic comparisons and $95 \% \mathrm{Cl}$ for pharmacodynamic comparisons), and expressed as percentages. A conclusion of no interaction was made if the entire $\mathrm{Cl}$ was within the standard no effect boundaries of $80-125 \%$. The Wilcoxon signed rank test was evaluated for the differences in $t_{\max }$ between treatment days for INR and R- and S-warfarin concentrations.

To assess the effect of concomitant warfarin dosing on ambrisentan pharmacokinetics, individual within-subject pair-wise differences of day 13 (warfarin + ambrisentan) $v s$. day 12 (ambrisentan alone) were summarized for the natural logarithms of ambrisentan $C_{\text {min,ss, }} C_{\text {max }, s s}$ and $A \cup C_{0-\tau, s s}$. These comparisons were reported as GMRs with $90 \% \mathrm{Cl}$ and expressed as percentages, and a conclusion of no interaction was made if the entire $\mathrm{Cl}$ was within the standard no effect boundaries of $80-125 \%$. The Wilcoxon signed rank test was evaluated for the differences in $t_{\max }$ between treatment days for ambrisentan concentrations.

A total of 22 subjects were enrolled to ensure that 18 subjects completed the study. With 18 subjects, the study had a $>80 \%$ power to detect a $6 \%$ difference in INR between day 13 (warfarin +ambrisentan) and day 1 (warfarin alone), and a $>90 \%$ probability that the true $95 \%$ twosided $\mathrm{Cl}$ for the comparison of day 13 and day 1 INR values was within $80-125 \%$. All statistical analyses were performed using the SAS ${ }^{\oplus}$ program (Cary, NC, USA).

\section{Results}

A total of 22 healthy male $(n=19,86.4 \%)$ and female $(n=3$, $13.6 \%)$ subjects with a mean age of $38.8 \pm 10.3$ years and mean weight of $79.1 \pm 10.4 \mathrm{~kg}$ were enrolled. All 22 enrolled subjects completed the study according to the protocol and received two doses of racemic warfarin $25 \mathrm{mg}$ and 12 doses of ambrisentan $10 \mathrm{mg}$; therefore, all were included in the safety, pharmacokinetic and pharmacodynamic assessments.

\section{Warfarin pharmacokinetics}

There were no clinically relevant effects of multiple doses of ambrisentan on the concentration-time profiles of Rand S-warfarin (Figure 2). Both R- and S-warfarin were detectable at the first postdose sampling time $(0.5 \mathrm{~h})$ and reached peak concentrations at approximately $1 \mathrm{~h}$ postdose. The concentration-time profiles of both warfarin enantiomers were virtually superimposable in the presence (day 13) or absence (day 1) of ambrisentan. Following multiple doses of ambrisentan, the observed mean $\mathrm{AUC}_{0 \text {-last }}$ values for R- and S-warfarin increased by $4.7 \%$ (GMR 104.7; $90 \% \mathrm{Cl} 101.7,107.7)$ and $1.6 \%(101.6 ; 90 \% \mathrm{Cl}$
98.4, 105.0), respectively, and mean $C_{\max }$ values decreased for both enantiomers by $8.4 \%(91.6 ; 90 \% \mathrm{Cl} 86.2,97.4)$ and $10.1 \%$ (93.0; $90 \% \mathrm{Cl} 90.8,95.3$ ), respectively (Table 1 ). The $90 \%$ Cls of the GMRs for R- and S-warfarin $C_{\max }$ were within the predefined $80-125 \%$ no effect boundaries. Furthermore, median $t_{\max }$ values for both enantiomers were comparable in the presence or absence of ambrisentan.

\section{Warfarin pharmacodynamics}

Administration of warfarin in the presence of multiple doses of ambrisentan (Figure 3) resulted in a $14.7 \%$ decrease in $I^{N} R_{\max }(85.3 ; 95 \% \mathrm{Cl} 82.4,88.2)$ and a $7.0 \%$ decrease in INR AuC(0-last) $(93.0 ; 95 \% \mathrm{Cl} 90.8,95.3)$. The $95 \% \mathrm{Cls}$ of the GMRs for these parameters were within the predefined $80-125 \%$ no effect boundaries (Table 2). No effect of ambrisentan on median $t_{\max }$ of INR was observed.

\section{Ambrisentan pharmacokinetics}

Racemic warfarin had little effect on the steady-state plasma concentration-time profile of ambrisentan (Figure 4). In the presence of warfarin, steady-state ambrisentan $C_{\max }$ and $C_{\min }$ decreased by $2.0 \%(98.0 ; 90 \% \mathrm{Cl}$ $87.6,109.5)$ and $9.3 \%(90.7 ; 90 \% \mathrm{Cl} 85.5,96.2)$, respectively (Table 3), and steady-state $A \cup C_{0-\tau}$ (where $\tau$ is the length of the $24-\mathrm{h}$ dosing interval) decreased by $12.2 \%(87.8 ; 90 \% \mathrm{Cl}$ $83.9,91.8)$. The $90 \% \mathrm{Cls}$ for all ambrisentan pharmacokinetic parameters were within the predefined no effect boundaries. Median $t_{\max }$ was comparable between day 12 and day 13.

\section{Safety and tolerability}

Thirteen participants (59.1\%) experienced an AE during the study. The most common AEs were headache, reported in seven (31.8\%) subjects, and dry skin and chapped lips, reported in three (13.6\%) subjects each. There were no serious AEs, and no subjects discontinued the study due to an AE. All reported AEs were mild in severity, and only one $A E$ of thirst was considered by the investigator to be probably related to study drug.

\section{Discussion}

In this study, multiple doses of ambrisentan $10 \mathrm{mg}$ once daily resulted in slight to modest changes in $\mathrm{R}$ - and $\mathrm{S}$-warfarin pharmacokinetic $\left(A \cup C_{0 \text {-last, }} C_{\text {max }}\right.$ ) and pharmacodynamic (INR) parameters. These changes were all within the predefined equivalence criteria of $80-125 \%$, suggesting that ambrisentan did not have a clinically relevant effect on warfarin metabolism or the resulting therapeutic effects of warfarin. Nonetheless, warfarin is a drug with a narrow therapeutic index, so that even slight changes must be considered in the context of clinical relevance.

Drug interactions with warfarin have been previously demonstrated for the ERAs bosentan and sitaxsentan [9, 14-16]. These interactions have been attributed to alter- 

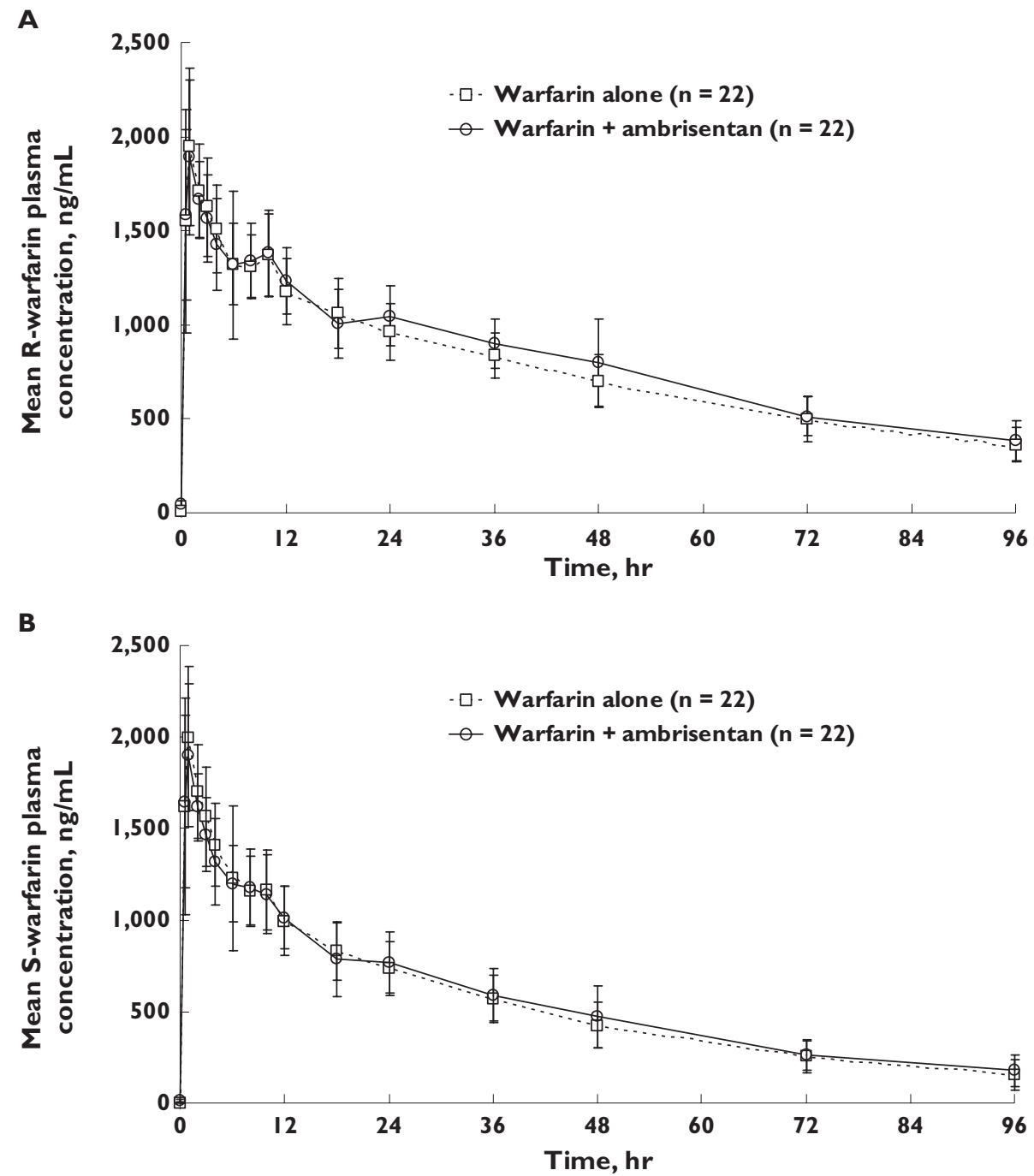

\section{Figure 2}

Mean plasma concentration vs. time profiles of R-warfarin (a) and S-warfarin (b) following a single oral dose of racemic warfarin $25 \mathrm{mg}$ alone ( $\square$ ) and in the presence of multiple doses of ambrisentan $(\bigcirc)$ in 22 healthy subjects. Data are means \pm standard deviation

ations in the activity of CYP2C9 and CYP3A4 for bosentan and CYP2C9 for sitaxsentan, and these two enzymes are also primarily responsible for metabolism of warfarin. Bosentan, which is primarily metabolized by CYP2C9 and CYP3A4, also induces the metabolic activity of these enzymes. As a result, co-administration of bosentan with racemic warfarin in healthy subjects decreased the systemic exposure (AUC) to the R- and S-enantiomers by 38 and $29 \%$, and these pharmacokinetic effects were associated with corresponding decreases in $\mathbb{I N R}_{\max }$ and $I_{\text {INR }}$ Auc(0-last) of 23 and $38 \%[14,15]$. However, clinical experience with concomitant administration of bosentan and warfarin in patients with PAH does not appear to result in any clinically relevant changes in INR or warfarin dose [17]. Sitaxsentan also has an interaction with warfarin due to sitaxsentan-mediated inhibition of CYP2C9 that increases the pharmacokinetic exposure of warfarin, prolonging its effects on coagulation [16]. The increase in INR observed with sitaxsentan co-administration requires substantial reduction in the daily dose of warfarin. For example, an $80 \%$ reduction in warfarin dose was required during the clinical studies of sitaxsentan to prevent excessive anticoagulation and the potential for serious bleeding episodes [16].

Ambrisentan is primarily metabolized via hepatic glucuronidation [10-12] and does not undergo significant phase I metabolism by CYP pathways. In addition, in vitro studies suggest that ambrisentan does not alter the expression or activity of CYP enzymes, thus minimizing the risk for drug-drug interactions with other agents metabolized by CYP family members. In this study, ambrisentan decreased INR $\max$ and INR $R_{\text {Auc(0-last) }}$ by 14.7 and $7.0 \%$, respectively, with a nominal increase in $\mathrm{AUC}_{0 \text {-last }}$ for $\mathrm{R}$ - and S-warfarin of 4.7 and $1.6 \%$, respectively. The mechanism of 


\section{Table 1}

Effect of ambrisentan on R- and S-warfarin pharmacokinetics

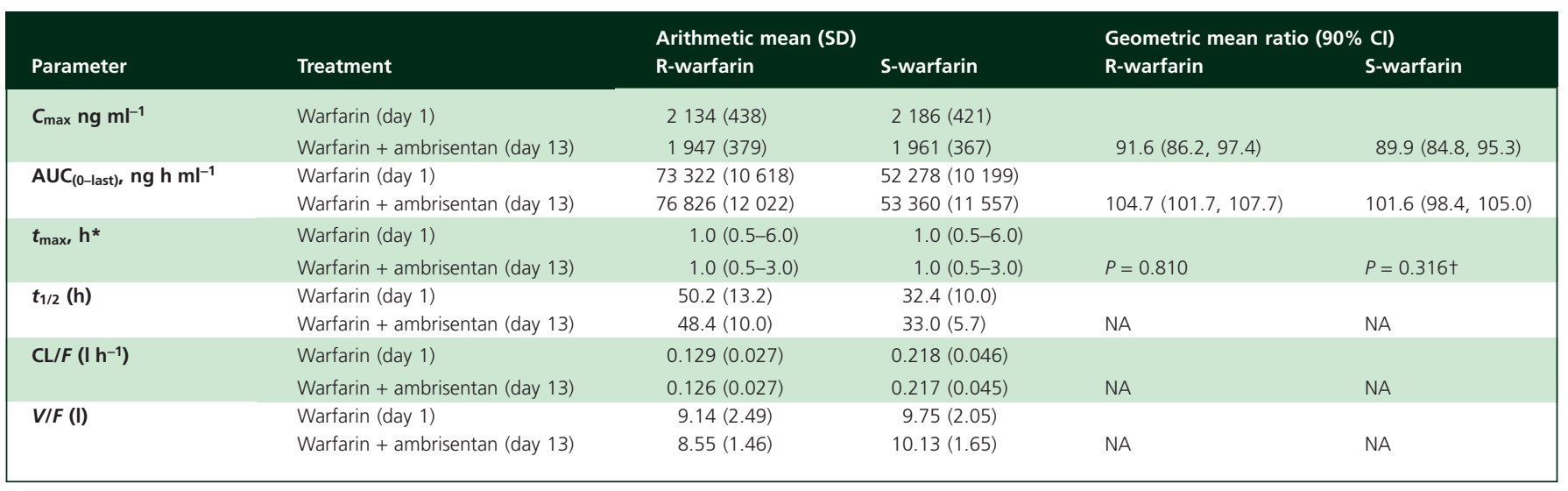

*Data are medians (minimum, maximum). $+P$-value of Wilcoxon signed rank test of hypothesis that median pair-wise day $13-$ day 12 difference $=0$. SD, standard deviation; $\mathrm{Cl}$, confidence intervals; $A \cup C_{(0-l a s t)}$, area under the plasma concentration-time curve from time 0 to last observation; $C_{\text {max }}$ maximum plasma concentration; $t_{\text {max }}$ time to reach maximum plasma concentration; $t_{1 / 2}$, terminal elimination half-life; CLF, clearance; VIF, volume of distribution; NA, not applicable.

\section{Table 2}

Effect of ambrisentan on warfarin pharmacodynamics

\begin{tabular}{|c|c|c|c|c|}
\hline Parameter & Treatment & $n$ & Arithmetic mean (SD) & Geometric mean ratio $(95 \% \mathrm{Cl})$ \\
\hline \multirow[t]{2}{*}{ INR $_{\max }$} & Warfarin (day 1) & 22 & $1.94(0.42)$ & \\
\hline & Warfarin + ambrisentan (day 13) & 22 & $1.65(0.31)$ & $85.3(82.4,88.2)$ \\
\hline \multirow[t]{2}{*}{ INR $_{\mathrm{AUC}(0-\text { last })}, \mathrm{h}^{-1}$} & Warfarin (day 1) & 22 & $143.9(21.1)$ & \\
\hline & Warfarin + ambrisentan (day 13) & 22 & $133.2(13.6)$ & $93.0(90.8,95.3)$ \\
\hline \multirow[t]{2}{*}{$\mathrm{INR}_{\mathrm{tmax}}, \mathrm{h}^{*}$} & Warfarin (day 1) & 22 & $36(24.0-48.0)$ & \\
\hline & Warfarin + ambrisentan (day 13) & 22 & $36(18.0-47.9)$ & $P=0.078 t$ \\
\hline
\end{tabular}

*Data are medians (minimum, maximum). $+P$-value of Wilcoxon signed rank test of hypothesis that median pair-wise day $13-$ day 12 difference $=0$. SD, standard deviation;

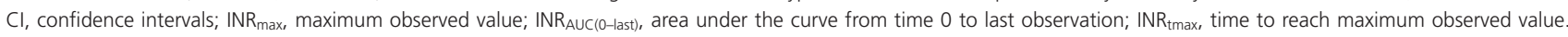

\section{Table 3}

Effects of racemic warfarin on ambrisentan steady-state pharmacokinetics

\begin{tabular}{|c|c|c|c|c|}
\hline Parameter & Treatment & $n$ & Arithmetic mean (SD) & Geometric mean ratio $(90 \% \mathrm{Cl})$ \\
\hline \multirow[t]{2}{*}{$C_{\text {max,ss }} \mathrm{ng} \mathrm{ml}^{-1}$} & Ambrisentan (day 12) & 22 & $988.6(278.3)$ & \\
\hline & Ambrisentan + warfarin (day 13) & 22 & $944.1(177.8)$ & $98.0(87.6,109.5)$ \\
\hline \multirow[t]{2}{*}{$C_{\min , s s} \mathrm{ng} \mathrm{ml}^{-1}$} & Ambrisentan (day 12) & 22 & $68.4(35.1)$ & \\
\hline & Ambrisentan + warfarin (day 13) & 22 & $60.5(25.8)$ & $90.7(85.5,96.2)$ \\
\hline \multirow[t]{2}{*}{$\operatorname{AUC}_{(0-\tau, s s)}, \mathrm{ng} \mathrm{h} \mathrm{ml}^{-1}$} & Ambrisentan (day 12) & 21 & $6384(2000)$ & \\
\hline & Ambrisentan + warfarin (day 13) & 22 & $5516(1609)$ & $87.8(83.9,91.8)$ \\
\hline \multirow[t]{2}{*}{$t_{\text {max }, s s} h^{*}$} & Ambrisentan (day 12) & 22 & $2.0(1.0-3.0)$ & \\
\hline & Ambrisentan + warfarin (day 13) & 22 & $2.00(1.0-3.0)$ & $P=0.957 \dagger$ \\
\hline \multirow[t]{2}{*}{$t_{1 / 2}(h)$} & Ambrisentan (day 12) & 21 & $8.3(1.9)$ & \\
\hline & Ambrisentan + warfarin (day 13) & 22 & $10.5(4.6)$ & NA \\
\hline \multirow[t]{2}{*}{$\mathrm{CL} / F\left(\mathrm{~h}^{-1}\right)$} & Ambrisentan (day 12) & 21 & $1.71(0.50)$ & \\
\hline & Ambrisentan + warfarin (day 13) & 22 & $1.96(0.55)$ & NA \\
\hline \multirow[t]{2}{*}{$V / F(I)$} & Ambrisentan (day 12) & 21 & $20.0(6.2)$ & \\
\hline & Ambrisentan + warfarin (day 13) & 22 & $29.1(12.8)$ & NA \\
\hline
\end{tabular}

*Data are medians (minimum, maximum). $+P$-value of Wilcoxon signed rank test of hypothesis that median pair-wise day $13-$ day 12 difference $=0$. SD, standard deviation; $\mathrm{Cl}$, confidence interval; $A \cup C_{(0-\tau)}$, area under the plasma concentration-time curve from time 0 to last observation; ss, steady state; $C_{\text {max, }}$ maximum plasma concentration; $C_{\text {min }}$, minimum plasma concentration; $t_{\max }$, time to reach maximum plasma concentration; $t_{1 / 2}$, terminal elimination half-life; $C L / F$, clearance; $V / F$, volume of distribution; NA, not applicable. 


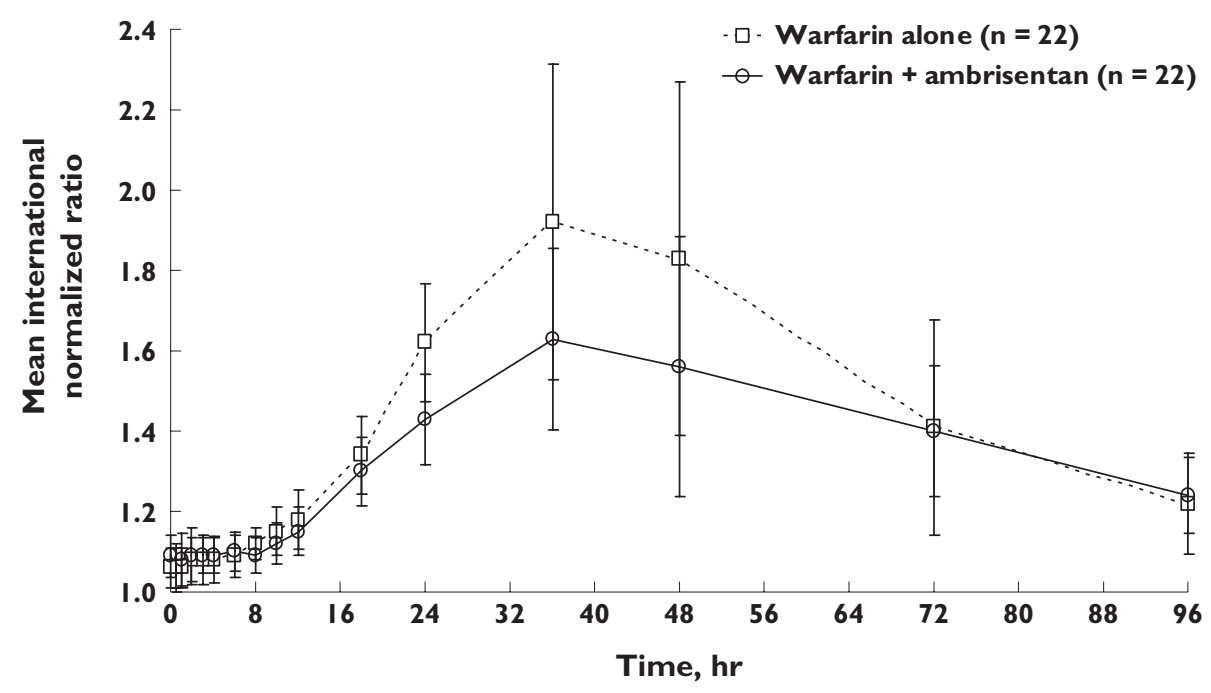

\section{Figure 3}

Mean effect vs. time profile of International Normalized Ratio following a single oral dose of $25 \mathrm{mg}$ racemic warfarin alone ( $\square$ ) and in the presence of multiple doses of ambrisentan $(O)$ in 22 healthy subjects. Data are means \pm standard deviation

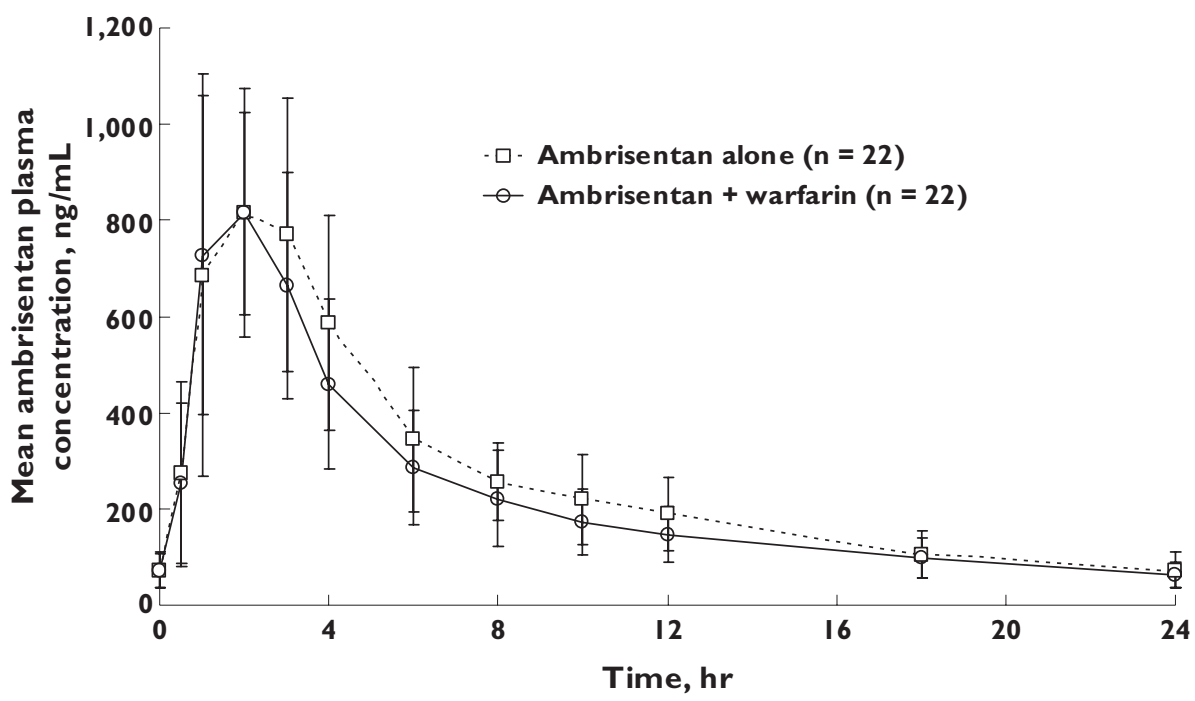

\section{Figure 4}

Mean plasma concentration-time profiles of ambrisentan following multiple doses of ambrisentan alone $(\square)$ and in the presence of a single dose of warfarin $(O)$ in 22 healthy subjects. Data are means \pm standard deviation

these minor interactions remains unknown, but is unlikely to be due to CYP inhibition or induction. Compared with bosentan, the smaller effects observed in this study are unlikely to result in any clinically relevant effects on warfarin dose or prothrombin time. The lack of a drug interaction between ambrisentan and warfarin has been corroborated in ambrisentan clinical studies that permitted the use of warfarin or warfarin-like anticoagulants as concomitant medications [10].

In this study, both ambrisentan and warfarin were administered at clinically effective doses, and no signifi- cant changes in the pharmacokinetics or pharmacodynamics of warfarin were observed with co-administration. However, because of the small sample size, short dosing duration and the use of healthy subjects rather than patients with $\mathrm{PAH}$, limited inference can be drawn from this study alone regarding the safety profile of chronic co-administration of ambrisentan and warfarin. Of note, clinical studies in patients with $\mathrm{PAH}$, such as those mentioned above, have demonstrated that chronic co-administration of ambrisentan and warfarin does not appear to be associated with any specific safety concerns 
or need for dose adjustment of either drug $[10,11]$. The results of this single-dose study in healthy volunteers are consistent with that conclusion. Nonetheless, warfarin is a drug with a narrow therapeutic window; therefore, caution should be exercised when initiating ambrisentan therapy in patients receiving warfarin.

\section{Competing interests}

This study was funded by Myogen Inc. (now Gilead Sciences Inc.). G.W., A.M. and C.D. are employees of Gilead Sciences Inc. J.V. is an independent consultant to Gilead Sciences Inc.

We would like to acknowledge the following individuals for their contributions to the completion of this study: M. J. Gerber (Formerly of Gilead Sciences Inc.), H. A. Ghofrani (University Hospital Giessen and Marburg, Germany), H. Pentikis and L. Zhu (ICON Development Solutions).

\section{REFERENCES}

1 Rubin LJ. Primary pulmonary hypertension. N Engl J Med 1997; 336: 111-7.

2 Farber HW, Loscalzo J. Pulmonary arterial hypertension. N Engl J Med 2004; 351: 1655-65.

3 Barst RJ, Rubin LJ, McGoon MD, Caldwell EJ, Long WA, Levy PS. Survival in primary pulmonary hypertension with long-term continuous intravenous prostacyclin. Ann Intern Med 1994; 121:409-15.

4 Humbert M, Sitbon O, Simmonneau G. Treatment of pulmonary arterial hypertension. N Engl J Med 2004; 351: 1425-36.

5 Takahashi H, Echizen H. Pharmacogenetics of warfarin elimination and its clinical implications. Clin Pharmacokinet 2001; 40: 587-603.

6 Chan E, McLachlan A, O'Reilly R, Rowland M. Stereochemical aspects of warfarin drug interactions: use of a combined pharmacokinetic-pharmacodynamic model. Clin Pharmacol Ther 1994; 56: 286-94.
7 Kaminsky LS, Zhang ZY. Human P450 metabolism of warfarin. Pharmacol Ther 1997; 73: 67-74.

8 Rubin LJ, Badesch DB, Barst RJ, Galie N, Black CM, Keogh A, Pulido T, Frost A, Roux S, Leconte I, Landzberg M, Simonneau $G$ for the Bosentan Randomized Trial of Endothlin Antagonist Therapy Study Group. Bosentan therapy for pulmonary arterial hypertension. N Engl J Med 2002; 346: 896-903.

9 Barst RJ, Langleben D, Frost A, Horn EM, Oudiz R, Shapiro S, McLaughlin V, Hill N, Tapson VF, Robbins IM, Zwicke D, Duncan B, Dixon RAF, Frumkin LR, the STRIDE-1 Study Group. Sitaxsentan therapy for pulmonary arterial hypertension. Am J Respir Crit Care Med 2004; 169: 441-7.

10 Galie N, Badesch D, Oudiz R, Simonneau G, McGoon MD, Keogh AM, Frost AE, Zwicke D, Naeije R, Shapiro S, Olschewski H, Rubin LJ. Ambrisentan therapy for pulmonary arterial hypertension. J Am Coll Cardiol 2005; 46: 529-35.

11 Rubin LJ, Dufton C, Gerber M. Ambrisentan for pulmonary arterial hypertension. Future Cardiol 2005; 1: 1-8.

12 Gilead Sciences I. Letairis (ambrisentan) tablets for oral use. United States Food and Drug Administration Drug Product Label, 6-15-2007.

13 Naidong W, Lee JW. Development and validation of a high-performance liquid chromatographic method for the quantitation of warfarin enantiomers in human plasma. J Pharm Biomed Anal 1993; 11: 785-92.

14 Weber C, Banken L, Birnboeck H, Schulz R. Effect of the endothelin-receptor antagonist bosentan on the pharmacokinetics and pharmacodynamics of warfarin. J Clin Pharmacol 1999; 39: 847-54.

15 Dingemanse J, Van Giersbergen PL. Clinical pharmacology of bosentan, a dual endothelin receptor antagonist. Clin Pharmacokinet 2004; 43: 1089-115.

16 Horn EM, Widlitz AC, Barst RJ. Sitaxsentan, a selective endothelin-A receptor antagonist for the treatment of pulmonary arterial hypertension. Expert Opin Investig Drugs 2004; 13: 1483-92.

17 Actelion Pharmaceuticals Ltd. Tracleer (bosentan) tablets. United States Food and Drug Administration Drug Product Label, 2006. 